\title{
Quantum limit in a quasi-one-dimensional conductor in a high tilted magnetic field
}

\author{
A.G. Lebed ${ }^{*}$ \\ Department of Physics, University of Arizona, 1118 E. 4-th Street, Tucson, AZ 85721, USA
}

\begin{abstract}
Recently, we have suggested Fermi-liquid - non-Fermi-liquid angular crossovers which may exist in quasi-one-dimensional (Q1D) conductors in high tilted magnetic fields [see A.G. Lebed, Phys. Rev. Lett. 115, 157001 (2015).] All calculations in the Letter, were done by using the quasiclassical Peierls substitution method, whose applicability in high magnetic fields was questionable. Here, we solve a fully quantum mechanical problem and show that the main qualitative conclusions of the above mentioned Letter are correct. In particular, we show that in high magnetic fields, applied along one of the two main crystallographic axis, we have $2 \mathrm{D}$ electron spectrum, whereas, for directions of high magnetic fields far from the axes, we have $1 \mathrm{D}$ electron spectrum. The later is known to promote non-Fermi-liquid properties. As a result, we expect the existence of Fermi-liquid - non-Fermi-liquid angular crossovers or phase transitions. Electronic parameters of Q1D conductor $(\mathrm{Per})_{2} \mathrm{Pt}(\mathrm{mnt})_{2}$ show that such transitions can appear in feasible high magnetic fields of the order of $H \simeq 20-25 T$
\end{abstract}

PACS numbers: 74.70.Kn, 71.10.Ay, 71.10.Hf, 75.20.En

Fermi-liquid theory has been successful in explanations of very unusual magnetic properties in quasi-onedimensional (Q1D) conductors [1]. The first historically discovered such properties, the so-called Field-Induced Spin-Density-Waves and related 3D quantum Hall effect in Q1D conductors (TMTSF $)_{2} \mathrm{X}\left(\mathrm{X}=\mathrm{ClO}_{4}, \mathrm{PF}_{6}, \mathrm{NO}_{3}\right.$, etc.) $[2,3]$, were explained $[1,4-10]$ by studying peculiarities of semiclassical electron motion along open Q1D sheets of the Fermi surface and electron-hole interactions under such conditions. New concept, $3 D \rightarrow 2 D$ dimensional crossover, was introduced [4] in the framework of Fermi-liquid approach. Extensions of this concept to different $3 D \rightarrow 1 D \rightarrow 2 D$ crossovers in a tilted magnetic field allowed to explain such phenomena as the socalled Lebed's magic angles (LMA) and Lee-NaughtonLebed's oscillations (see, for example, Ref.[11] and references therein). It is important that all the above mentioned physical phenomena happen in low enough magnetic fields, where the "sizes" of electron trajectories are less than inter-chain and inter-plane distances. Despite the above-described success, non-Fermi-liquid properties have been also studied both theoretically [12-15] and experimentally. For instance, there exist experimental claims that Fermi liquid cannot explain adequately the LMA phenomena in the Nernst [16-19] and Hall [20] effects.

An important step in studying magnetic properties of layered Q1D compounds was made in Ref.[21], where it was suggested that some other types of $3 D \rightarrow 2 D$ crossovers - quantum dimensional crossovers - can occur in such compounds in high magnetic fields. It was shown [21] that, at high magnetic fields, the typical "sizes" of electron trajectories can become less than inter-layered distances in layered Q1D conductors, which results in the appearance of such unusual phenomenon as the Reentrant Superconductivity [21-23]. Different types of quantum $3 D \rightarrow 2 D$ and $3 D \rightarrow 1 D$ dimensional crossovers have been recently studied theoretically [24-27]. In particular, in Ref.[26], it was shown that, when strong magnetic field was applied far enough from the main crystallographic axes of a Q1D conductor, we have almost $1 D$ electron spectrum and can expect non-Fermi-liquid behavior, whereas, for the magnetic field directions close to the main crystallographic axis, we have $2 \mathrm{D}$ electron spectrum and, thus, Fermi-liquid properties have to restore. On this basis, in Ref.[26], Fermi-liquid - non-Fermi-liquid angular crossovers were suggested to occur in a Q1D conductor in a high magnetic field and the critical magnetic field was estimated as $H \simeq 25 T$ in layered Q1D conductor $(\mathrm{Per})_{2} \mathrm{Pt}(\mathrm{mnt})_{2}$.

We pay attention that all previous considerations of the quantum dimensional crossovers [21-27] are based on quasi-classical version of the so-called Peierls substitution method $[4,1]$. The goal of our paper is to solve a fully quantum mechanical problem for a Q1D geometry of electron spectrum in a perpendicular magnetic field. We show that at high magnetic fields electron wave functions indeed localize on the 1D conducting chains, unless the magnetic field is directed along one of the main crystallographic axes. If a magnetic field is directed along one of the axes, it is shown that electron wave functions are localized only on 2D planes. This property of electron wave functions confirms the hypothesis of Ref.[26] about possible angular Fermi-liquid - non-Fermi-liquid crossovers (or phase transitions).

At first, let us consider a $3 D$ isotropic electron spectrum,

$$
\epsilon\left(p_{x}, p_{y}, p_{z}\right)=\frac{p_{x}^{2}}{2 m}+\frac{p_{y}^{2}+p_{x}^{2}}{2 m}
$$

where $\frac{p_{x}^{2}}{2 m}$ is electron energy along the conducting chains, in the following magnetic field, inclined perpendicular to the chains:

$$
\mathbf{H}=(0, H \sin \alpha, H \cos \alpha),
$$




$$
\mathbf{A}=(H z \sin \alpha-H y \cos \alpha, 0,0) .
$$

It is important that electron motion along the chains is free and characterized by the large energies, $\frac{p_{x}^{2}}{2 m} \sim \epsilon_{F}$, where $\epsilon_{F}$ is the Fermi energy. Therefore, the Peierls substitution method for momentum $p_{x}$ in Eq.(1) is an exact procedure, $p_{x} \rightarrow-i \frac{\partial}{\partial x}-(e / c) A_{x}$, where $\hbar \equiv 1$. As a result, we obtain for kinetic energy in magnetic field (2) the following operator:

$$
\begin{aligned}
\hat{\epsilon}(x, y, z)= & \frac{1}{2 m}\left[\left(-i \frac{\partial}{\partial x}+\frac{e H y \cos \alpha}{c}-\frac{e H z \sin \alpha}{c}\right)^{2}\right. \\
& \left.+\left(-i \frac{\partial}{\partial y}\right)^{2}+\left(-i \frac{\partial}{\partial y}\right)^{2}\right]
\end{aligned}
$$

As seen from Eq.(3), the magnetic field (2) does not disturb electron motion along the chains, where electrons are characterized by conserved momenta close to the Fermi momentum, $p_{F}$. Therefore, we represent electron wave functions in the following way:

$$
\Psi_{\epsilon}(x, y, z)=\exp \left(i p_{x} x\right) \Psi_{\epsilon}(y, z), \quad p_{x} \simeq p_{F} .
$$

Substitution of the wave function (4) into the kinetic energy operator (3) results in

$$
\begin{aligned}
\hat{\epsilon}(y, z)= & \frac{1}{2 m}\left[\left(p_{F}+\frac{e H y \cos \alpha}{c}-\frac{e H z \sin \alpha}{c}\right)^{2}\right. \\
& \left.+\left(-i \frac{\partial}{\partial y}\right)^{2}+\left(-i \frac{\partial}{\partial y}\right)^{2}\right] .
\end{aligned}
$$

Note that below we keep in electron energy (5) only terms of the order of $\omega_{b}(\alpha)$ and $\omega_{c}(\alpha)$ and disregard terms of the order of $\omega_{b}^{2}(\alpha) / \epsilon_{F} \ll \omega_{b}(\alpha)$ and $\omega_{c}^{2}(\alpha) / \epsilon_{F} \ll \omega_{c}(\alpha)$, where

$$
\omega_{b}(\alpha)=\frac{e H v_{F} b^{*} \cos \alpha}{c}, \quad \omega_{c}(\alpha)=\frac{e H v_{F} c^{*} \sin \alpha}{c}
$$

are the so-called cyclotron frequencies [26]. Here, $b^{*}$ and $c^{*}$ are the crystalline lattice parameters along $\mathbf{y}$ and $\mathbf{z}$ axes, correspondingly; $v_{F}=p_{F} / m$ is the Fermi velocity along the conducting $\mathbf{x}$ axis. Therefore, we can linearize kinetic energy operator (5) with respect to the frequencies, $\omega_{b}(\alpha)$ and $\omega_{c}(\alpha)$ :

$$
\begin{aligned}
& \frac{1}{2 m}\left(p_{F}+\frac{e H y \cos \alpha}{c}-\frac{e H x \sin \alpha}{c}\right)^{2} \\
& =\frac{1}{2 m}\left[p_{F}+\frac{\omega_{b}(\alpha)}{v_{F}} \frac{y}{b^{*}}-\frac{\omega_{c}(\alpha)}{v_{F}} \frac{z}{c^{*}}\right]^{2} \\
& \simeq \epsilon_{F}+\omega_{b}(\alpha) \frac{y}{b^{*}}-\omega_{c}(\alpha) \frac{z}{c^{*}} .
\end{aligned}
$$

If electron potential energy in $(\mathbf{y}, \mathbf{z})$ plane, perpendicular to the conducting axis, is $V(y, z)$, then the corresponding Schrödinger equation for electron wave functions can be written as

$$
\begin{array}{r}
{\left[-\frac{1}{2 m}\left(\frac{\partial^{2}}{\partial y^{2}}+\frac{\partial^{2}}{\partial z^{2}}\right)+\omega_{b}(\alpha) \frac{y}{b^{*}}-\omega_{c}(\alpha) \frac{z}{c^{*}}\right.} \\
+V(y, z)] \Psi_{\tilde{\epsilon}}(y, z)=\tilde{\epsilon} \Psi_{\tilde{\epsilon}}(y, z),
\end{array}
$$

where $\tilde{\epsilon}=\epsilon-\epsilon_{F}$. Below, we consider the following model for the in-plane potential energy,

$V(y, z)=-\frac{\kappa_{1}}{m} \sum_{n_{1}=-\infty}^{+\infty} \delta\left(y-n_{1} b^{*}\right)-\frac{\kappa_{2}}{m} \sum_{n_{2}=-\infty}^{+\infty} \delta\left(z-n_{2} c^{*}\right)$

where $\delta(y)$ and $\delta(z)$ are the 1D Dirac delta-functions. In this case, as we show below, a tight-binding variant of the Scrödinger equation in a magnetic field (8),(9) becomes exactly solvable. In particular, the total $2 \mathrm{D}$ wave function in Eq.(8) can be factorized in our case:

$$
\Psi(y, z)=\Psi_{\tilde{\epsilon}}(y) \Psi_{\tilde{\epsilon}}(z)
$$

For 1D wave functions (10), it is easy to obtain the following Schrödinger equations:

$$
\begin{gathered}
{\left[-\frac{1}{2 m}\left(\frac{d^{2}}{d y^{2}}\right)+\omega_{b}(\alpha) \frac{y}{b^{*}}-\frac{\kappa_{1}}{m} \sum_{n_{1}=-\infty}^{+\infty} \delta\left(y-n_{1} b^{*}\right)\right]} \\
\times \Psi_{\tilde{\epsilon}_{1}}(y)=\tilde{\epsilon}_{1} \Psi_{\tilde{\epsilon}_{1}}(y)
\end{gathered}
$$

and

$$
\begin{gathered}
{\left[-\frac{1}{2 m}\left(\frac{d^{2}}{d z^{2}}\right)-\omega_{c}(\alpha) \frac{z}{b^{*}}-\frac{\kappa_{2}}{m} \sum_{n_{2}=-\infty}^{+\infty} \delta\left(z-n_{2} c^{*}\right)\right]} \\
\times \Psi_{\tilde{\epsilon}_{2}}(z)=\tilde{\epsilon}_{2} \Psi_{\tilde{\epsilon}_{2}}(z)
\end{gathered}
$$

where $\tilde{\epsilon}=\tilde{\epsilon}_{1}+\tilde{\epsilon}_{2}$.

Let us first consider Eq.(11) for $y$ coordinate. To solve Eq.(11), we use the so-called tight-binding approximation, where the wave function can be expressed as

$$
\Psi_{\tilde{\epsilon}_{1}}(y)=\sum_{m=-\infty}^{+\infty} A_{m}(H, \alpha) \phi_{\epsilon_{01}}\left(y-m b^{*}\right) .
$$

Here, the wave function,

$$
\phi_{\epsilon_{01}}(y)=\sqrt{\kappa_{1}} \exp \left(-\kappa_{1}|y|\right), \quad \epsilon_{01}=-\frac{\kappa_{1}^{2}}{2 m}
$$

is solution of the following equation:

$$
\left[-\frac{1}{2 m}\left(\frac{d^{2}}{d y^{2}}\right)-\frac{\kappa_{1}}{m} \delta(y)\right] \phi_{\epsilon_{01}}(y)=\epsilon_{01} \phi_{\epsilon_{01}}(y) .
$$

By using the tight-binding approximation, it is possible to show that the amplitudes $A_{m}(H, \alpha)$ in Eq.(13) satisfy the following equation:

$$
\begin{gathered}
A_{m}(H, \alpha)\left[\tilde{\epsilon}_{1}-\epsilon_{01}-m \omega_{b}(\alpha)\right] \\
+A_{m+1}(H, \alpha) t_{1}+A_{m-1}(H, \alpha) t_{1}=0
\end{gathered}
$$

where

$$
t_{1}=\frac{\kappa_{1}}{m} \phi_{\epsilon_{01}}(0) \phi_{\epsilon_{01}}\left( \pm b^{*}\right)=\frac{\kappa_{1}^{2}}{m} \exp \left(-\kappa_{1} b^{*}\right) .
$$


Now, we consider Eq.(12) for $z$ coordinate. As it was done above, to solve Eq.(12), we use the tight-binding approximation, where the wave function (12) can be written as

$$
\Psi_{\tilde{\epsilon}_{2}}(z)=\sum_{l=-\infty}^{+\infty} B_{l}(H, \alpha) \phi_{\epsilon_{02}}\left(z-l c^{*}\right)
$$

where the wave function,

$$
\phi_{\epsilon_{02}}(z)=\sqrt{\kappa_{2}} \exp \left(-\kappa_{2}|z|\right), \quad \epsilon_{02}=-\frac{\kappa_{2}^{2}}{2 m},
$$

is solution of the equation:

$$
\left[-\frac{1}{2 m}\left(\frac{d^{2}}{d y^{2}}\right)-\frac{\kappa_{2}}{m} \delta(z)\right] \phi_{\epsilon_{02}}(z)=\epsilon_{02} \phi_{\epsilon_{02}}(z) .
$$

In the same way as for the amplitudes $A_{m}(H, \alpha)$ in Eq.(13), it is possible to show that the amplitudes $B_{l}(H, \alpha)$ in Eq.(18) satisfy the following equation:

$$
\begin{gathered}
B_{l}(H, \alpha)\left[\tilde{\epsilon}_{2}-\epsilon_{02}+l \omega_{c}(\alpha)\right] \\
+B_{l+1}(H, \alpha) t_{2}+B_{l-1}(H, \alpha) t_{2}=0,
\end{gathered}
$$

where

$$
t_{2}=\frac{\kappa_{2}}{m} \phi_{\epsilon_{02}}(0) \phi_{\epsilon_{02}}\left( \pm c^{*}\right)=\frac{\kappa_{2}^{2}}{m} \exp \left(-\kappa_{2} c^{*}\right) .
$$

We pay attention that Eqs. (16) and (21) for the amplitudes, $A_{m}(H, \alpha)$ and $B_{l}(H, \alpha)$, are similar, although the cyclotron frequencies (6) have different signs in Eqs.(16) and (21).

To solve Eqs.(16) and (21), we make use of the following properties of the Bessel functions [28]:

$$
z J_{n-1}(z)+z J_{n+1}(z)=2 n J_{n}(z),
$$

where $J_{n}(z)$ is the Bessel function of the $\mathrm{n}$-th order. To this end, we rewrite Eqs.(16) and (21) in the similar ways:

$$
\frac{2 t_{1}}{\omega_{b}(\alpha)} A_{m-1}+\frac{2 t_{1}}{\omega_{b}(\alpha)} A_{m+1}=2\left[m-\frac{\epsilon_{1}-\epsilon_{0}}{\omega_{b}(\alpha)}\right] A_{m}
$$

and

$$
\frac{2 t_{2}}{\omega_{c}(\alpha)} B_{l-1}+\frac{2 t_{2}}{\omega_{c}(\alpha)} B_{l+1}=2\left[m-\frac{\epsilon_{1}-\epsilon_{0}}{\omega_{c}(\alpha)}\right] B_{l} .
$$

Comparing Eqs.(24) and (25) with Eq.(23) for the Bessel functions, we can conclude that Eqs.(24) and (25) have the following solutions:

$$
\tilde{\epsilon}_{1}=\epsilon_{01}+n_{1} \omega_{b}(\alpha), \quad \tilde{\epsilon}_{2}=\epsilon_{02}-n_{2} \omega_{c}(\alpha)
$$

and

$$
A_{m}(H, \alpha)=J_{m-n_{1}}\left[\frac{2 t_{1}}{\omega_{b}(\alpha)}\right]
$$

$$
B_{l}(H, \alpha)=J_{n_{2}-l}\left[\frac{2 t_{2}}{\omega_{c}(\alpha)}\right],
$$

where $n_{1}$ and $n_{2}$ are some quantum numbers.

Therefore, electron wave functions (13) and (18) along $\mathbf{y}$ and $\mathbf{z}$ axes, respectively, can be rewritten as

$$
\Psi_{\tilde{\epsilon}_{1}}(y)=\sum_{m=-\infty}^{+\infty} J_{m-n_{1}}\left[\frac{2 t_{1}}{\omega_{b}(\alpha)}\right] \phi_{\epsilon_{01}}\left(y-m b^{*}\right) .
$$

and

$$
\Psi_{\tilde{\epsilon}_{2}}(z)=\sum_{l=-\infty}^{+\infty} J_{n_{2}-l}\left[\frac{2 t_{2}}{\omega_{c}(\alpha)}\right] \phi_{\epsilon_{02}}\left(z-l c^{*}\right),
$$

where the total energy is

$$
\epsilon=\epsilon_{F}+\epsilon_{01}+\epsilon_{02}+n_{1} \omega_{b}(\alpha)-n_{2} \omega_{c}(\alpha) .
$$

From Eqs.(29) and (30), it directly follows that the electron wave function is centered in the $n_{1}$-th conducting chains along $\mathbf{y}$ axis and along the $n_{2}$-th conducting chains along $\mathbf{z}$ axis, respectively. Now, let us consider physical properties of wave functions (29) and (30) for different directions and strengths of a magnetic field. Suppose that a magnetic field is applied far from the main crystallographic axis,

$$
|\alpha| \sim 1, \quad|\alpha-\pi / 2| \sim 1,
$$

then at high enough magnetic fields,

$$
H \gg \frac{2 t_{1} c}{e v_{F} b^{*} \cos \alpha}, \quad H \gg \frac{2 t_{2} c}{e v_{F} c^{*} \sin \alpha},
$$

arguments of the Bessel functions in Eqs. (29) and (30) are small, $2 t_{1} / \omega_{b}(\alpha) \ll 1$ and $2 t_{2} / \omega_{c}(\alpha) \ll 1$. As it follows, from the theory of the Bessel functions (see, for example, [28]), the all Bessel functions with the exceptions of that with $m=n_{1}$ and $l=n_{2}$ in Eqs.(29) and (30) are small and, thus, the wave functions can be written in this case approximately as

$$
\Psi_{\tilde{\epsilon}_{1}}(y) \approx \phi_{\epsilon_{01}}\left(y-n_{1} b^{*}\right) .
$$

and

$$
\Psi_{\tilde{\epsilon}_{2}}(z) \approx \phi_{\epsilon_{02}}\left(z-n_{2} c^{*}\right) .
$$

Note that the localization of the wave functions (34) and (35) on one Q1D chain promotes non-Fermi-liquid properties. On the other hand, if magnetic field direction is close enough to one of the main crystallographic axes, corresponding in our case to

$$
\alpha_{1}=0, \quad \alpha_{2}=\pi / 2,
$$

then even in high magnetic fields (33) one of the Bessel functions in electron wave functions (29) and (30) becomes delocalized [28]. The latter means that the Fermiliquid properties have to restore for angles (36). Therefore, we can conclude that at high enough magnetic fields 
of the order of

$$
H \geq H^{*}=\max \left[\frac{2 \sqrt{2} t_{1} c}{e v_{F} b^{*}}, \quad \frac{2 \sqrt{2} t_{2} c}{e v_{F} c^{*}}\right]
$$

there have to be angular crossover (or phase transition) between Fermi-liquid and non-Fermi-liquid states. [Note that in Eq.(37) we have put $\alpha=\pi / 4$.] It is important that wave functions (29) and (30), derived in this paper by a full quantum mechanical method, are similar to the quasi-classical wave functions (9) of Ref.[26]. We pay also attention to the fact that the derived in this paper equation for the corresponding critical field, $H^{*}$, coincides with Eq.(12) from Ref.[26]. Therefore, we make a statement that we justify hypothesis about the angular Fermi-liquid - non-Fermi-liquid crossover (or phase transitions) suggested in Ref.[26]. In particular, the corresponding critical magnetic field for Q1D organic material (Per $)_{2} \mathrm{Pt}(\mathrm{mnt})_{2}$ under pressure can be estimated as $H^{*} \simeq 25 T[26]$.

In the paper, we have calculated fully quantum mechanical wave function for the case, where a Q1D conductor is placed in a tilted perpendicular magnetic field. We have evaluated energy in a magnetic field with accuracy $\omega_{b}(\alpha) \sim \omega_{c}(\alpha)$ and disregarded only terms of the order of $\omega_{b}^{2}(\alpha) / \epsilon_{F} \sim \omega_{c}^{2}(\alpha) / \epsilon_{F}$. As a result, we reproduced major results of Ref.[26], where the so-called quasiclassical Peierls substitution method was used. Our conclusion is that the Peierls substitution methods is adequate not only for quasi-classical dimensional crossovers [4-14], where the "sizes" of electron orbits are larger than inter-chain and inter-plane distances, but also for quantum dimensional crossovers [21-27], where the "sizes' of the orbits are less than inter-chain and inter-plane distances, In some sense, in this paper we have validated previously obtained well-known results [4-14], [21-27], and some others.

We are thankful to N.N. Bagmet, D. Graf, N.E. Hussey, and J. Singleton for useful discussions. ${ }^{*}$ Also at: L.D. Landau Institute for Theoretical Physics, RAS, 2 Kosygina Street, Moscow 117334, Russia.

[1] The Physics of Organic Superconductors and Conductors, edited by A.G. Lebed (Springer, Berlin, 2008).

[2] P.M. Chaikin, Mu-Yong Choi, J.F. Kwak, J.S. Brooks, K.P. Martin, M.J. Naughton, E.M. Engler, and R.L. Green, Phys. Rev. Lett. 51, 2333 (1983).

[3] M. Ribault, D. Jerome, J. Tuchendler, C. Weyl, and K. Bechgaard, J. Phys. (Paris) Lett. 44, L953 (1983).

[4] L.P. Gorkov and A.G. Lebed, J. Phys. (Paris) Lett. 45, L433 (1984).

[5] M. Heritier, G. Montambaux, and P. Lederer, J. Phys. (Paris) Lett. 45, L943 (1984).

[6] P.M. Chaikin, Phys. Rev. B 31, 4770 (1985).

[7] A.G. Lebed, Sov. Phys. JETP, 62, 595 (1985) [Zh. Eksp. Teor. Fiz. 89, 1034 (1985)].

[8] A. Virosztek, L. Chen, and K. Maki, Phys. Rev. B 34, 3371 (1986).

[9] D. Poilblanc, M. Heritier, G. Montambaux, and P. Lederer, J.Phys. C Solid State Phys. 19, L321 (1986).

[10] V.M. Yakovenko, Phys. Rev. B 43, 11353 (1991)].

[11] A.G. Lebed and Si Wu, Phys. Rev. B 82, 172504 (2010).

[12] A.G. Lebed and Per Bak, Phys. Rev. Lett. 63, 1315 (1989).

[13] V.M. Yakovenko, Phys. Rev. Lett., 68, 3607 (1992); Erratum 70, 519 (1993).

[14] A.G. Lebed,

[15] S.P.Strong, D.G. Clark, and P.W. Anderson, Phys. Rev. Lett. 73, 1007 (1994).

[16] W. Wu, I.J. Lee and P.M. Chaikin, Phys. Rev. Lett. 91, 056601 (2003).

[17] W. Wu, N.P. Ong, and P.M. Chaikin, Phys. Rev. B 72, 235116 (2005).

[18] E.S. Choi, J.S. Brooks, H. Kang, Y.J. Jo, and W. Kang, Phys. Rev. Lett., 95, 187001 (2005).

[19] M.-S. Nam, A. Ardavan, W. Wu, and P.M. Chaikin, Phys. Rev. B 74, 073105 (2006).

[20] K. Kobayashi, H. Satsukawa, J. Yamada, T. Terashita, and S. Uji, Phys. Rev. Lett. 112, 116805 (2014).

[21] A.G. Lebed, JETP Lett. 44, 114 (1986) [Pis'ma Zh. Eksp. Teor. Fiz. 44, 89 (1986)].

[22] N. Dupuis, G. Montambaux, and C.A.R. Sa de Melo, Phys. Rev. Lett. 49, 8993 (1994)].

[23] A.G. Lebed and K. Yamaji, Phys. Rev. Lett. 80, 2697 (1998).

[24] O. Sepper and A.G. Lebed, Phys. Rev. B 90, 094509 (2014).

[25] A.G. Lebed and O. Sepper, Phys. Rev. B 90, 024510 (2014).

[26] A.G. Lebed, Phys. Rev. Lett. 115, 157001 (2015).

[27] A.G. Lebed, Phys. Rev. B 94, 035162 (2016).

[28] I.S. Gradshteyn and I.M. Ryzhik, Table of Integrals, Series, and Products, 5th edition (Academic Press, New York, 1994). 